\title{
A Study of the Influence of New Generation Granulated Sorbents on the Processes Regulating the Aggregate State of the Blood with the Use of Piezoelectric Thromboelastography
}

\author{
Sergey V. Barannikov ${ }^{1}, \mathrm{PhD}$; Evgeniy F. Cherednikov ${ }^{1}, \mathrm{PhD}$, ScD; Igor S. Yuzefovich ${ }^{1}, \mathrm{PhD}$; \\ Ludmila E. Mehantjeva ${ }^{1}, \mathrm{PhD}, \mathrm{ScD}$; Igor F. Filev², PhD; Yuri V. Maleev ${ }^{3}, \mathrm{PhD}, \mathrm{ScD}$; \\ Evgeniy S. Ovsyannikov ${ }^{1 *}, \mathrm{PhD}, \mathrm{ScD}$; Violetta A. Maslova ${ }^{1}$ \\ ${ }^{1}$ Voronezh State Medical University named after N. N. Burdenko \\ ${ }^{2}$ Voronezh City Clinical Emergency Hospital № 1 \\ ${ }^{3}$ Voronezh Basic Medical College \\ Voronezh, the Russian Federation
}

\begin{abstract}
Background: Gastroduodenal bleeding (GDB) and the improvement of endoscopic hemostasis (EH) remain a priority in emergency surgery. This article presents the results of an experimental study of the effects of granular sorbents (Aseptisorb, Aseptisorb-A, Aseptisorb-D) on the system regulating the aggregate state of the blood using modern capabilities of piezoelectric thromboelastography (TEG).

Methods and Results: The study involved 12 healthy volunteers (9/75\% men and 3/25\% women) aged between 18 and 58 years, with the average age of $34.0(26.0 ; 44.0)$ years.

For the study, the blood of healthy volunteers with normal indicators of the system regulating the aggregate state of the blood was used. In vitro experiments: Several tests were performed with the blood of each volunteer. In the first experiment (the control stage), the blood cuvette did not contain the test material. At the second stage of the experiment, the hemostatic properties of new generation granulated sorbents (Aseptisorb, Aseptisorb-A, and Aseptisorb-D) were studied.

Experimental studies have shown that the use of granular sorbents Aseptisorb, Aseptisorb-A, and Aseptisorb-D in varying degrees affects the links of platelet and coagulation hemostasis, providing acceleration of thrombosis processes while increasing the maximum density of the clot. These effects determine the effectiveness of the clinical use of these sorbents to stop various types of bleeding.

Conclusion: Experimental studies of the effect of granular sorbents on the system regulating the aggregate state of the blood using piezoelectric TEG have shown that the use of Aseptisorb, Aseptisorb-A, and Aseptisorb-D can significantly reduce the time of blood clotting and increase the maximum clot density, which determines the possibility of the use of these sorbents in endoscopic hemostatic treatment for GDB. (International Journal of Biomedicine. 2021;11(3):286-290.)
\end{abstract}

Key Words: piezoelectric thromboelastography $\bullet$ the aggregate state of the blood $\bullet$ granular sorbents

For citation: Barannikov SV, Cherednikov EF, Yuzefovich IS; Mehantjeva LE, Filev IF, Maleev YuV, Ovsyannikov ES, Maslova VA. A Study of the Influence of New Generation Granulated Sorbents on the Processes Regulating the Aggregate State of the Blood with the Use of Piezoelectric Thromboelastography. International Journal of Biomedicine. 2021;11(3):286-290. doi:10.21103/ Article11(3)_OA6

\section{Abbreviations}

CPC, contact phase of coagulation; EH, endoscopic hemostasis; GDB, gastroduodenal bleeding; TEG, thromboelastography; U, units.

\section{Introduction}

The problem of bleeding and the improvement of surgical hemostasis methods has remained a priority in emergency surgery for many decades. One of the most technically complex types of hemostasis is endoscopic arrest of Gastroduodenal bleeding (GDB).$^{(1-6)}$ Modern endoscopy has many ways to stop bleeding, among which the most common are coagulation 
methods (argonplasma coagulation, diathermocoagulation, laser photocoagulation, etc.), injection hemostasis, clipping, and application methods, as well as combined techniques. Even so, the rate of recurrence of hemorrhage, even after successful primary endoscopic hemostasis $(\mathrm{EH})$, reaches 10\%-46\%, which shows the need to improve the capabilities of therapeutic endoscopy. ${ }^{(7-11)}$

Methods of EH by insufflation of powdered hemostatic systems, such as Hemospray, EndoClot, etc., to the source of hemorrhage are becoming increasingly widespread globally in clinical practice. The main disadvantage of such systems is their high cost, which limits the possibility of their use in everyday clinical practice. ${ }^{(12,13)}$ It should be noted that using powdered hemostatic systems in therapeutic endoscopy is not new. For more than 25 years, granular sorbents with hemostatic, antibacterial, and other properties have been successfully used for endoscopic hemostasis of gastroduodenal bleeding. However, the mechanism of action of these sorbents on the system of regulation of the aggregate state of blood remains not fully understood..$^{(14-17)}$

The aim of our research was to study the peculiarities of the influence of new generation granular sorbents on the system regulating the aggregate state of the blood through in vitro experiments using the modern possibilities of piezoelectric TEG.

\section{Materials and Methods}

The study involved 12 healthy volunteers $(9 / 75 \%$ men and $3 / 25 \%$ women) aged between 18 and 58 years, with the average age of $34.0(26.0 ; 44.0)$ years.

For the study, the blood of healthy volunteers with normal indicators of the system regulating the aggregate state of the blood was used. In the aseptic conditions, venous blood was collected with a venipuncture needle into vacuum tubes containing a $3.8 \%$ sodium citrate solution with a volume of $4.5 \mathrm{ml}$, intended for coagulographic studies. The contents of the test tube were carefully mixed by tilting the test tube several times.

The study of the parameters of the regulation of the aggregate state of the blood was performed using the piezoelectric thromboelastograph ARP-01M "Mednord."

In vitro experiments: Several tests were performed with the blood of each volunteer. In the first experiment (the control stage), the blood cuvette did not contain the test material. Next, $0.3 \mathrm{ml}$ of citrate blood was added to the TEG cuvette using a laboratory single-channel pipette dispenser, then the blood cuvette was placed in the device's thermostat chamber and a coagulation activator solution $(0.025 \mathrm{M}$ calcium chloride solution) was added. The sensor of the device was immersed in the cuvette and the study was started.

At the second stage of the experiment, the hemostatic properties of new generation granulated sorbents (Aseptisorb, Aseptisorb-A, and Aseptisorb-D) were studied. To do this, $1.0 \mathrm{mg}$ of the sorbent was added to the cuvette of the device filled with $0.3 \mathrm{ml}$ of citrate blood. The powdered sorbent was evenly mixed with the test blood, then the activator solution was added and the study was started.
The results of the studies were analyzed by evaluating the following parameters of thromboelastograms: the time of the $\mathrm{CPC}$, the intensity of the $\mathrm{CPC}$, the time to reach the thrombin constant, the constant of thrombin activity, the time of blood clotting, the intensity of the coagulation drive, the time of clot polymerization, the intensity of clot polymerization, the time of fibrin-platelet clot formation, the maximum density of the clot, the intensity of total clotting, and the time of the beginning of fibrinolysis, according to the device instructions. ${ }^{(18-19)}$

The study was conducted in accordance with ethical principles of the WMA Declaration of Helsinki (1964, ed. 2013) and approved by the Ethics Committee of Voronezh State Medical University named after N. N. Burdenko. Written informed consent was obtained from all participants.

Statistical analysis was performed using Microsoft Excel software package. For descriptive analysis, results are presented as median $(\mathrm{Me})$, lower quartile $\left(\mathrm{Q}_{1}\right)$ and upper quartile $\left(\mathrm{Q}_{\mathrm{u}}\right)$. A non-parametric Kruskal-Wallis test was used for comparisons of median values among four groups, followed by post-hoc testing using un-paired Mann-Whitney U tests.

\section{Results and Discussion}

When analyzing thromboelastograms of healthy volunteers, it was found that the time of the CPC was $1.0(1.0 ; 1.0) \mathrm{min}$, while the intensity of the CPC was at the level of $39.5(17.7 ; 63.0) \mathrm{U}$ (Table 1$)$. The time to reach the thrombin constant occurred at $5.9(4.6 ; 7.0) \mathrm{min}$, and the constant of thrombin activity was at the level of $17.8(13.6 ; 27.4) \mathrm{U}$. Blood clotting in healthy individuals occurred at $12.1(10.7 ; 14.1) \mathrm{min}$, with the intensity of the coagulation drive of $19.3(10.8 ; 21.9)$ U. Clot polymerization occurred at $23.5(20.7 ; 25.3) \mathrm{min}$, and the intensity of clot polymerization was $13.1(5.3 ; 14.5)$ U. Formation of the fibrin-platelet clot was observed at $32.6(29.9 ; 38.8) \mathrm{min}$. The maximum clot density in healthy individuals was $389.0(289.0 ; 444.5) \mathrm{U}$ with the total clotting intensity at $7.9(6.1 ; 11.8) \mathrm{U}$. At the same time, it should be noted that in 3 observations, the clot lysis process began at 29.7(29.5;29.9)min.

\section{Aseptisorb effects}

When studying the effect of Aseptisorb on the dynamics of the processes regulating the aggregate state of the blood, we found no significant changes in the time of the CPC compared to the control stage. The time of the CPC for Aseptisorb was $1.0(1.0 ; 1.0) \mathrm{min}$, but the intensity of the CPC was at a higher level and amounted to $80.5(70.0 ; 108.5) \mathrm{U}(P=0.002)$. Under the influence of Aseptisorb, the time to reach the thrombin constant occurred earlier than in the control stage $(P=0.003)$. At the same time, the constant of thrombin activity was also more pronounced, 54.1(25.9;62.5)U $(P=0.002)$. The use of Aseptisorb significantly reduced the blood clotting time from $12.1(10.7 ; 14.1) \mathrm{min}$ to $6.8(5.6 ; 8.8) \mathrm{min}(P=0.000)$ and increased the intensity of the coagulation drive from $19.3(10.8 ; 21.9) \mathrm{U}$ to $34.6(16.5 ; 41.3) \mathrm{U}(P=0.03)$. The onset of clot polymerization occurred at an earlier time than in the control stage $[(16.8(15.6 ; 18.8) \mathrm{min}$ and $23.5(20.7 ; 25.3)$ min, respectively, $P=0.000)]$, but the intensity of clot 
polymerization did not differ significantly from the control stage. A fibrin-platelet clot was formed at 28.1(24.3;29.7) min versus $32.6(29.9 ; 38.83) \mathrm{min}$ in the control stage $(P=0.04)$. Analyzing the characteristics of the clot density, we found that due to the sorbtion activity of Aseptisorb and its hydrophilic properties, the maximum clot density was higher than in the control stage $[(468.5(438.0 ; 696.5) \mathrm{U}$ and $389.0(289.0 ; 444.5) \mathrm{U}$, respectively, $P=0.005)$ ], as was the intensity of total clotting $[(12.3(10.6 ; 19.8) \mathrm{U}$ and 7.9 $(6.1 ; 11.8) \mathrm{U}$, respectively, $P=0.005)]$. Clot lysis in the use of Aseptisorb was not observed in any study.

\section{Aseptisorb-A effects}

Analyzing the effect of Aseptisorb-A on the parameters of the regulation of the blood aggregate state, we found that, like Aseptisorb, Aseptisorb-A had no effect on the time of the CPC. The time of the CPC for Aseptisorb was $1.0(1.0 ; 1.0) \mathrm{min}$. However, the use of Aseptisorb-A allowed an increase in the intensity of the CPC to $99.5(67.0 ; 158.5)$ $\mathrm{U}$ from $39.5(17.3 ; 63.0) \mathrm{U}$ in the control stage $(P=0.001)$. For Aseptisorb-A, the time to reach the thrombin constant was $3.3(2.2 ; 4.6) \mathrm{min}$, and the thrombin activity constant was 58.3(30.8;97.2)U. The use of Aseptisorb-A reduced the blood

Table 1

The influence of new generation granular sorbents on TEG parameters

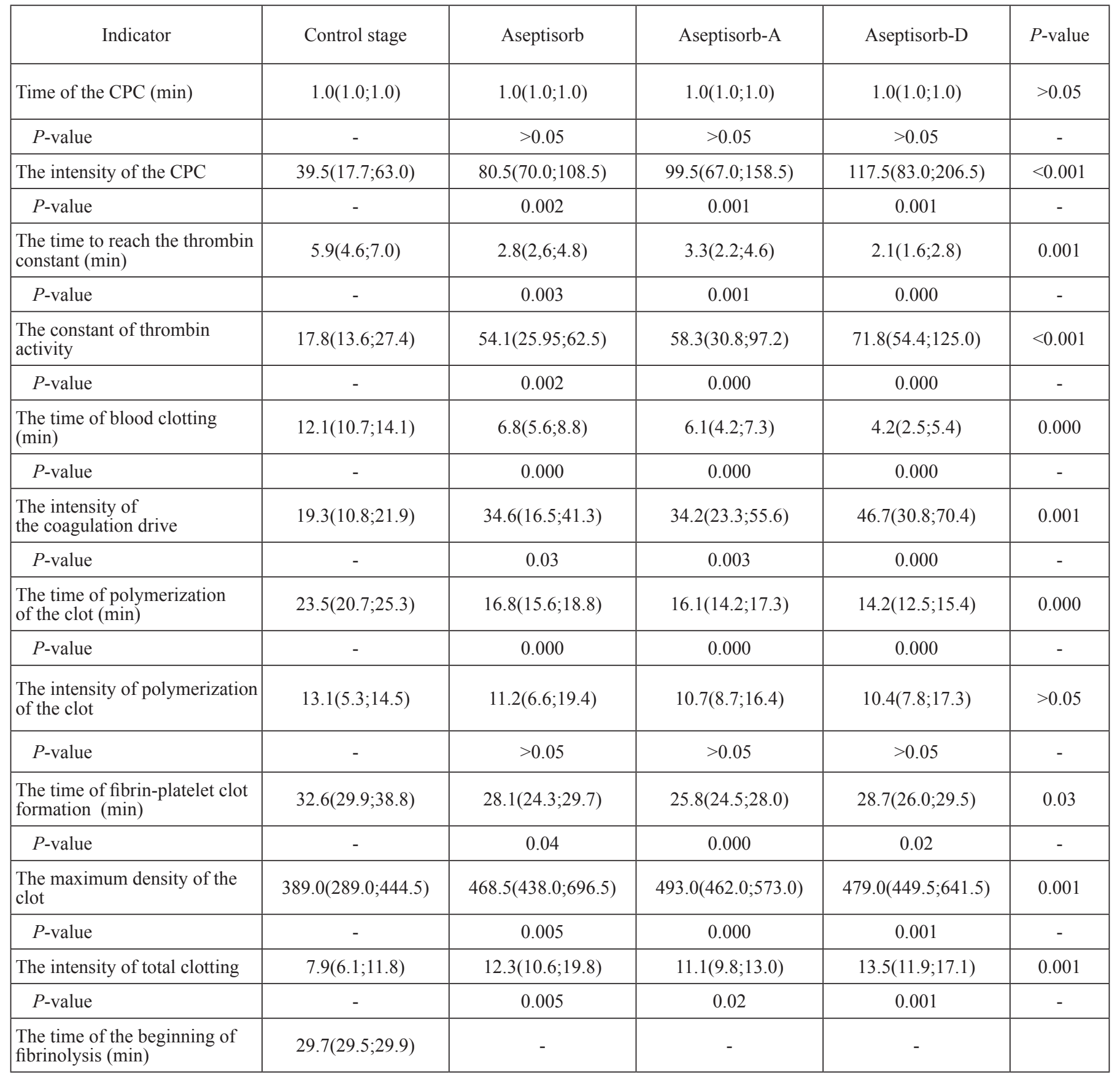


clotting time from $12.1(10.7 ; 14.1) \mathrm{min}$ to $6.1(4.2 ; 7.3) \mathrm{min}$ $(P=0.000)$ and simultaneously increased the intensity of the coagulation drive from $19.3(10.8 ; 21.9) \mathrm{U}$ to $34.2(23.3 ; 55.6)$ $\mathrm{U}(P=0.003)$. Clot polymerization occurred earlier than in the control stage [(16.1(14.2;17.3)min and 23.5(20.7;25.3)min, respectively, $P=0.000)]$, but the intensity of clot polymerization did not differ significantly from the control stage. The use of this sorbent also reduced the time of formation of a fibrinplatelet clot (from $32.6(29.9 ; 38.8 \mathrm{~min}$ to $25.8(24.5 ; 28.0) \mathrm{min}$, $P=0.000$ ), while increasing the maximum clot density (from $389.0(289.0 ; 444.5) \mathrm{U}$ to $493.0(462.0 ; 573.0) \mathrm{U}, P=.000)$. At the same time, the use of Aseptisorb-A allowed an increase in the intensity of total coagulation (from $7.9(6.1 ; 11.8) \mathrm{U}$ to 11.1(9.8;13.0) $\mathrm{U}, P=0.02)$. An increase in the density of the clot with Aseptisorb-A contributed to the formation of a stable clot, and the phenomena of fibrinolysis were not observed.

\section{Aseptisorb-D effects}

The influence of Aseptisorb-D on the dynamics of the processes of regulation of the blood aggregate state also had distinctive features. Thus, the time of the CPC was at the level of $1.0(1.0 ; 1.0) \mathrm{min}$ and did not differ from the control stage. The intensity of the CPC was $117.5(83.0 ; 206.5) \mathrm{U}$ versus $39.5(17.3 ; 63.0) \mathrm{U}$ in the control stage $(P=0.001)$. The time to reach the thrombin constant was statistically reduced, compared to the control stage $[(2.1(1.6 ; 2.8) \mathrm{min}$ and $5.9(4.6 ; 7.0) \mathrm{min}$, respectively, $P=0.000)]$, and the thrombin activity constant was at a higher level than the control stage $[(71.8(54.5 ; 125.0)$ $\mathrm{U}$ and $17.8(13.6 ; 27.4) \mathrm{U}$, respectively, $P=0.000)$. The use of Aseptisorb-D significantly reduced the blood clotting time (from 12.1(10.7;14.1)min to $4.2(2.5 ; 5.4) \mathrm{min}, P=0.000)$, while the intensity of the coagulation drive was $46.7(30.8 ; 70.4) \mathrm{U}$. Clot polymerization occurred earlier than in the control stage $[14.2(12.5 ; 15.4) \mathrm{min}$ and $23.5(20.7 ; 25.3) \mathrm{min}$, respectively, $P=0.000)$ ], but the intensity of clot polymerization did not differ significantly from the control stage. The use of Aseptisorb-D reduced the time of formation of a fibrin-platelet clot (from $32.6(29.9 ; 38.8) \mathrm{min}$ to $28.7(26.0 ; 29.5) \mathrm{min}, \quad P=0.02)$, while simultaneously making it possible to increase the maximum clot density (from $389.0(289.0 ; 444.5) \mathrm{U}$ to $479.0(449.5 ; 641.5)$ $\mathrm{U}, P=0.001$ ) and total coagulation intensity (from $7.9(6.1 ; 11.8$ ) to $13.5(11.9 ; 17.1) \mathrm{U}, P=0.001)$. When using Aseptisorb-D, the clot was also dense and the processes of fibrinolysis were not recorded.

Summing up the results of the experimental study, the most important finding for surgeons is that among all indicators of blood clotting processes there are 2 main ones: the time of blood clotting and the maximum density of the clot. Blood clotting time is a key indicator that reflects the transition of the liquid state of the blood to a gel-like state and coincides with the implementation of the thrombin explosion and is highly correlated with the time of reaching the peak concentration of thrombin in the thrombin generation test. The maximum density is the final qualitative characteristic of the entire process of thrombosis and reflects the resistance of the clot to external influences. ${ }^{(2-24)}$ The use of the granulated sorbents Aseptisorb, Aseptisorb-A and Aseptisorb-D allows us to significantly accelerate the blood clotting time and increase the maximum clot density, which provides a theoretical justification for the effectiveness of these sorbents for surgical hemostasis.

\section{Conclusion}

Experimental studies have shown that the use of granular sorbents Aseptisorb, Aseptisorb-A, and Aseptisorb-D in varying degrees affects the links of platelet and coagulation hemostasis, providing acceleration of thrombosis processes while increasing the maximum density of the clot. These effects determine the effectiveness of the clinical use of these sorbents to stop various types of bleeding.

Experimental studies of the effect of granular sorbents on the system regulating the aggregate state of the blood using piezoelectric thromboelastography have shown that the use of Aseptisorb, Aseptisorb-A, Aseptisorb-D, and Sephadex G-50 can significantly reduce the time of blood clotting and increase the maximum clot density, which determines the possibility of the use of these sorbents in endoscopic hemostatic treatment for GDB.

\section{Competing Interests} interests.

The authors declare that they have no competing

\section{Sources of Funding}

This work was supported by the Council on Grants of the President of the Russian Federation for State Support of Young Scientists and Leading Scientific Schools (Grant MK1069.2020.7).

\section{References}

1. Cherednikov EF, Barannikov SV, Yuzefovich IS, Polubkova GV, Maleev YuV, Volkova IV, Vysotskaya AT, Strygin OV, Ovsyannikov ES. Innovative Endoscopic Technologies in the Complex Treatment of Patients with Unstable Stopped Gastroduodenal Bleeding. International Journal of Biomedicine. 2021;11(1):24-28. doi:10.21103/ Article11(1)_OA4

2. Budnevsky AV, Cherednikov EF, Popov AV, Ovsyannikov ES, Kravchenko AY, Fursov KO. A Complex Multidisciplinary Approach to Prevention Gastro-duodenal Bleeding in Patients of General Hospital. International Journal of Biomedicine. 2017;7(3):204-207. doi: 10.21103/Article7(3)_OA8

3. Cherednikov EF, Barannikov SV, Romantsov MN, Popov AV. New aspects of preventive endoscopic hemostasis in the treatment of peptic ulcer bleeding in the experimental condition. The EPMA Journal. 2017; 8(S1):45.

4. Cherednikov E F, Chernyh AV, Maleev YuV, Popov AV, Stekolnikov VV. [Topographical and anatomical prerequisites for the development of Mallory-Weiss syndrome]. Bulletin of

*Corresponding author: Evgeniy S. Ovsyannikov, PhD, ScD. Department of faculty therapy, Voronezh State Medical University named after N.N. Burdenko.Voronezh, Russia. E-mail: ovses@ yandex.ru 
the Russian Military Medical Academy. 2015;(S2):153-154. [Article in Russian].

5. Baev VE, Kravets BB, Cherednikov EF. [Diagnostics of ulcerative forms of stomach cancer]. Voronezh. 2003:112. [In Russian].

6. Cherednikov EF, Barannikov SV, Zhdanov AI, Moshurov IP, Polubkova GV, Maleev YuV, Ovsyannikov ES, Myachina DS. Combimed Use of Biologically Active Hemostatic and Granulated Sorbent in Endoscopic Cytoprotective Hemostasis in Patients with Bleeding Gastroduodenal Ulcers. International Journal of Biomedicine. 2020;10(2):129-132. doi: 10.21103/ Article10(2)_OA8

7. Cherednikov EF, Glukhov AA, Romantsov MN, Maleev YuV, Barannikov SV, Shkurina IA, Vysotskaya AT, Ovsyannikov ES. Hemostatic Agents in Combination with Diovine for Local Treatment of Simulated Bleeding Gastric Ulcers. International Journal of Biomedicine. 2020;10(2):138141. doi: 10.21103/Article10(2) OA10

8. Cherednikov EF, Budnevsky AV, Popov AV, Fursov KO. A new opinion on gastroduodenal bleeding preventon in patients with somatic pathology. The EPMA Journal. 2017;8(S1):46.

9. Romantsov MN, Cherednikov E F, Danilenko VI, Stepanov DS, Fursov K O, Deryaeva AG. [Morphological Characteristics of Processes of Simulated Bleeding Gastric Defects Reparation in Treatment with Gelplastan and Diovin]. Journal of Anatomy and Histopathology. 2017;6(1):81-86. [Article in Russian].

10. Cherednikov EF, Deryaeva OG, Adianov VV, Ovchinnikov IF, Popov AV. [Modern directions of prevention and treatment of patients with gastrointestinal bleeding in the center]. System Analysis and Management in Biomedical Systems. 2014;13(2):426-433. [Article in Russian].

11. Cherednikov EF, Maleev YuV, Chernyh AV, Litovkina TE, Cherednikov EE, Shevtsov AN. [Modern views on the etiology and pathogenesis of ruptured hemorrhagic syndrome (Mallory-Weiss syndrome)]. Journal of anatomy and Histopathology. 2016;5(1):86-98. [Article in Russian].

12. Cherednikov EF, Barannikov SV, Glukhov AA, Banin IN, Maleev YuV, Adianov VV. [The use of Aseptisorb-A and platelet-rich plasma in the complex endoscopic treatment of patients with ulcerative gastroduodenal bleeding]. Bulletin of Experimental and Clinical Surgery.2017;10(2):116-122. [Article in Russian].

13. Vitali F, Naegel A, Atreya R, Zopf S, Neufert C, Siebler J, Neurath MF, Rath T. Comparison of Hemospray ${ }^{\circledR}$ and Endoclot ${ }^{\mathrm{TM}}$ for the treatment of gastrointestinal bleeding. World J Gastroenterol. 2019 Apr 7;25(13):1592-1602. doi: 10.3748/wjg.v25.i13.1592.

14. Cherednikov EF, Maleev YuV, Chernyh AV, Litovkina TE, Bondarenko AA, Cherednikov EE, Popov AV. [Current views on the diagnosis, treatment, and prevention of ruptured hemorrhagic syndrome (Mallory-Weiss syndrome)]. Journal of New Medical Technologies. 2016;23(4):161-172. [Article in Russian].

15. Adianov VV, Cherednikov EF. [Optimizing treatment of gastroduodenal bleeding in patients with high surgical risk]. System Analysis and Management in Biomedical Systems. 2014;13(4):841-846. [Article in Russian].

16. Deryaeva OG, Cherednikov EF. [Multimodality therapy of erosiveulcer gastroduodenal bleeding by patients in a multidisciplinary hospital]. System Analysis and Management in Biomedical Systems. 2014; 13(3):725-730. [Article in Russian]. 17. Cherednikov EF, Batkaev AR, Baev VE. The Reparative regeneration of erosive-ulcerative lesions of the stomach and duodenum in the local treatment of hydrophilic granular sorbents. System Analysis and Management in Biomedical Systems. 2005;4(2):224-225. [Article in Russian].

18. Low-frequency piezoelectric thromboelastography in the diagnosis of hemostatic disorders: A methodological guide. Tomsk. 2018:35. [In Russian].

19. Hardware and software complex for clinical and diagnostic studies of the rheological properties of blood ARP-01M «Mednord»: Technical description and operating instructions. Tomsk. 2018:29. [In Russian].

20. Cherednikov EF, Barannikov SV, Maleev YuV, Fursov KO, Litovkina TE, Zakurdaev EI, Ovsyannikov ES. Experimental justification of using Aseptisorb-A and platelet-rich plasma in endoscopic treatment of mold bleeding stomach defects. International Journal of Biomedicine. 2017;7(4):298-301. doi: 10.21103/Article7(4)_OA5

21. Cherednikov EF, Kashurnikova MA, Romantsov MN, Barannikov SV, Bolkhovitinov A E, Gaponenkov DG, Lyubimov PYu. [Experimental study of new means of local hemostasis in the treatment of ulcerative bleeding]. Scientific and Medical Bulletin of the Central Chernozem Region. 2016;(65):27-33. [Article in Russian].

22. Cherednikov EF, Barannikov SV, Fursov KO, Polubkova GV, Danilenko VI, Stepanov DS. [Healing of bleeding experimental defects of the stomach with topical anilovin and platelet-rich plasma]. Journal of Volgograd State Medical University. 2017;2(62):130-133. [Article in Russian].

23. Cherednikov EF, Barannikov SV, Maleev YuV, Fursov $\mathrm{KO}$, Litovkina TE, Zakurdaev EI. [Experimental justification of the use of biologically active draining sorbent and plasma enriched by thrombocytes in treatment of bleeding defects of the stomach]. Journal of New Medical Technologies. 2017;24(2):114-118. [Article in Russian].

24. Tyurin II, Udut VV. Low-frequency piezothromboelastography of whole blood: algorithms for the diagnosis and correction of hemostatic disorders.Tomsk: Publishing House of Tomsk State University. 2016:170. [In Russian]. 